\title{
Vague Information Processing
}

\author{
Andreas Meier $\cdot$ Rudolf Seising
}

(C) Springer Fachmedien Wiesbaden GmbH, ein Teil von Springer Nature 2018

Im Zeitalter von Big Data werden immense Informationsbestände aus unterschiedlichen Quellen gesammelt: Mitteilungen (Postings) aus sozialen Netzwerken, elektronische Briefe und Korrespondenz, Anfragen aus Suchmaschinen, Dokumentensammlungen aus Content-Management-Systemen, Sensordaten beliebiger Art, Kursentwicklungen von Börsenplätzen, Daten aus Verkehrsströmen, Satellitenbilder, Videoaufnahmen, Messdaten von Geräten des Haushalts (Smart Meter), Kauf- und Bestellvorgänge aus elektronischen Shops, Daten aus eHealth-Anwendungen oder Aufzeichnungen von elektronischen Monitoring-Systemen.

Wie steht es mit der Qualität und der Aussagekraft dieser Daten? Das ursprüngliche ,3-V-Modell“, das Doug Laney im Jahre 2001 zur Fassung von Big Data einführte (Volume, Variety, Velocity), wurde nach und nach um weitere ,V's“ ergänzt: Value, Veracity, Validity, Volatility, Vulnerability oder Viability. Validity betrifft die Aussagekraft, Veracity steht mehr für Wahrhaftigkeit bzw. Glaubwürdigkeit der Daten.

Damit wird schon klar: Daten bzw. daraus abgeleitete Informationen sind oft missverständlich, unbestimmt, ungenau, ungewiss, unsicher oder vage. Deshalb drängt es sich auf, Verfahren zu entwickeln, welche die Ungewissheit der Daten miteinbeziehen und bewerten. Ein Lösungsansatz besteht darin, die unscharfe Logik des Berkeley-Professors Lotfi A. Zadeh (1921-2017) zur Bewertung der Information anzuwenden. Fuzzy Logic ist in gewissem Sinne eine Erweiterung der klassischen Logik, denn sie kennt nicht nur die beiden Wahrheitswerte wahr (1) und falsch (0),

\footnotetext{
A. Meier $(\bowtie)$

Universität Fribourg, Boulevard de Pérolles 90, 1700 Fribourg, Schweiz

E-Mail: andreas.meier@unifr.ch

R. Seising

Deutsches Museum München, Museumsinsel 1, 80538 München, Deutschland

E-Mail: r.seising@deutsches-museum.de
} 
sondern alle Abstufungen oder Grautöne dazwischen. In der Fuzzy Set Theorie erlaubt das Arbeiten mit Mengenzugehörigkeitsfunktionen deshalb, differenzierte Aussagen über die Qualität der Daten zu erhalten.

Im vorliegenden Schwerpunktheft HMD 321 über Vague Information Processing finden Sie zwei Überblicksbeiträge: Der erste beleuchtet die Entstehungsgeschichte der unscharfen Logik, verfasst vom Gastherausgeber Rudolf Seising, einem Wissenschafts- und Technikhistoriker am Deutschen Museum in München. Der zweite Beitrag von Edy Portmann geht auf das Anwendungsfeld Soft Computing näher ein und erläutert dies anhand der Entwicklung von Smart Cities.

Der Eröffnungsbeitrag zum Schwerpunkt widmet sich dem Design Thinking mit dem Ziel, durch ein Video das kreative Potenzial von Computing with Words potenziellen Nutzern aufzuzeigen. Es folgen Beiträge zum unscharfen Portfolio Management, zur Steigerung von Kooperation in virtuellen Teams sowie zur Predictive Maintenance in der Industrie.

Eine Verallgemeinerung der unscharfen Logik stellt die Intuitionistic Fuzzy Logic dar, die auf den Arbeiten von Krassimir Atanassov beruht. Neben der Mengenzugehörigkeitsfunktion $\mu(\mathrm{x})$ für Elemente $\mathrm{x}$ aus einer klassischen Menge X führt der Bulgare die sogenannte non-membership function $v(\mathrm{x})$ ein und bezeichnet die Differenz $1-\mu(x)-v(x)$ als ,,degree of indeterminacy“ (Unbestimmtheit). Roland Schütze von der IBM Deutschland und Hansjörg Fromm vom Karlsruhe Institute for Technology verwenden Intuitionistic Fuzzy Sets, um das Service Management in den Griff zu bekommen.

Der Umgang mit unsicheren Daten wird an zwei attraktiven Anwendungsbeispielen illustriert. In einer ersten Untersuchung werden verschiedene Algorithmen verglichen, um Duplikate von Musikwerken aus Big Data extrahieren zu können. Im zweiten Anwendungsfall für unsichere Daten hilft das Projekt Open Street Map, Standortentscheide für Filialen besser zu fällen.

Ein Beitrag zur politischen Partizipation mit einem Privacy Setting Framework sowie mit Hinweisen zum Politcontrolling und Public Memory rundet den Schwerpunkt ab.

Liebe HMD-Leserinnen und -Leser: Mit unserem Schwerpunktheft Vague Information Processing geben wir Ihnen einen Einblick in die Vielfalt der Methoden und Anwendungsoptionen bei unsicheren Daten. Eines ist sicher: Der Drang nach Qualitätssicherung bei Big Data Anwendungen bleibt auch in Zukunft ungebrochen. 